\title{
Factores de riesgo de colonización por Enterococcus spp resistente a vancomicina en pacientes pediátricos hospitalizados con patología oncológica
}

\author{
Paula Loyola, Juan Tordecilla, Dona Benadof, Karla Yohannessen y Mirta Acuña
}

\section{Risk factor of intestinal colonization with vancomycin resistant Enterococcus spp in hospitalized pediatric patients with oncological disease}

Background: The isolation of vancomycin-resistant Enterococcus spp (ERV) has increased significantly within the last few years, along with the risk of infection and dissemination of these bacteria. Our aim was to determine risk factors (RF) for intestinal colonization in hospitalized pediatric patients with oncological disease at Hospital de Niños Roberto del Río. Methods: Between January 2012 and December 2013 a transversal study was performed with 107 rectal swabs and processed with a PCR for ERV. The patients were classified as "colonized with ERV" and "not colonized with ERV" and we evaluated possible RF for intestinal colonization in both groups. Results: VRE colonization was found in 51 patients (52\%). The median of time elapsed between oncological diagnosis and VRE colonization was 35 days. The significant RF associated with VRE colonization were days of hospitalization prior to study, neutropenia and treatment with antibiotics within 30 days prior to study and mucositis. Conclusions: According to the RF revealed in this study we may suggest prevention standards to avoid ERV colonization. This is the first investigation in our country in hospitalized pediatric patients with oncological disease and processed with a multiplex PCR for ERV, therefore it is a great contribution about this subject in Chile.

Key words: Vancomycin-resistant Enterococcus spp, colonization, pediatric oncology.

Palabras clave: Enterococcus spp resistente a vancomicina, colonización, oncología pediátrica.

\section{Introducción}

L a resistencia bacteriana a diversos antimicrobianos es un problema creciente en el mundo. Enterococcus spp resistentes a vancomicina (ERV) fueron descritos por primera vez en Europa en el año 1986 y su aislamiento ha presentado un incremento significativo en el tiempo ${ }^{1}$. En el año 2009, 14 a 25\% de todos los Enterococcus spp pesquisados en los hospitales de Norteamérica eran ERV ${ }^{2}$. En nuestro país, en el año 2008 se describió que $15,9 \%$ de los estudios de colonización intestinal tomados en adultos hospitalizados en la Unidad de Cuidados Intensivos y de Quimioterapia del Hospital Militar estaban positivos para ERV 3 .

El mecanismo de acción de vancomicina es la inhibición de los estadios tardíos de la síntesis de la pared celular al unirse a precursores de peptidoglicanos. La resistencia de Enterococcus spp está dada por la alteración de los precursores de peptidoglicanos, los que tienen menor afinidad por este antimicrobiano ${ }^{1}$. Los ERV pueden expresar seis fenotipos: VanA, VanB, VanC, VanD, VanE y $\mathrm{VanG}^{4}$. El fenotipo VanA es resistente a vancomicina y teicoplanina, el fenotipo VanB es resistente a vancomicina y sensible a teicoplanina, el fenotipo VanC, presente en el ADN de E. gallinarum y E. casseliflavus, tiene baja resistencia a vancomicina y es sensible a teicoplanina ${ }^{5}$. Las infecciones en humanos están dadas principalmente por las especies E. faecalis y E. faecium, que pueden expresar el fenotipo VanA o VanB, el cual está contenido en material genético presente en plásmidos y por lo tanto, con alto riesgo de diseminación a otros Enterococcus spp sensibles a vancomicina ${ }^{4}$.

En la medida que aumenta la tasa de colonización por ERV aumenta el riesgo de infección. La asociación colonización-infección está dada principalmente por las características del hospedero; en una experiencia, 30\% de los pacientes inmunocomprometidos colonizados por ERV desarrollaron bacteriemia por este agente, con los riesgos y limitación de opciones terapéuticas que esto implica ${ }^{6}$. Por ello, resulta de gran importancia identificar a los pacientes colonizados por ERV, y mejor aún, identificar pacientes que están en riesgo de adquirir ERV y mejorar las condiciones que sean posibles para evitar que se conviertan en portadores de esta bacteria. La literatura científica sobre factores de riesgo (FR) en pacientes oncológicos pediátricos no es muy extensa, y no existen estudios en estos pacientes en nuestro país. El objetivo de este trabajo es determinar los FR de colonización
Universidad de Chile.

Facultad de Medicina, Departamento de Pediatría, Programa de Formación en Hemato-Oncología (PL). Escuela de Salud Pública (KY). Hospital de Niños Roberto del Río.

Servicio de Pediatría

Unidad de Onco-Hematología (JT).

Unidad de Infectología (MA).

Laboratorio de Microbiología (DB).

Establecimiento: Hospital de Niños Roberto del Río.

Los autores declaramos no tener conflictos de interés.

Fuente de financiamiento: Ninguna.

Recibido: 25 de septiembre de 2014

Aceptado: 19 de mayo de 2015

Correspondencia a:

Mirta Acuña Ávila

mirta.i.acuna@gmail.com 
intestinal por ERV en pacientes internados con patología oncológica pediátrica en el Hospital de Niños Roberto del Río (HRRío).

\section{Pacientes y Método}

\section{Centro asistencial}

El HRR es un centro que cuenta con 75 camas básicas, 11 de unidad de oncología y 49 camas críticas (UCI-UTIcardiocirugía). Recibe derivación de patología oncológica de la red PINDA (Programa Infantil Nacional de Drogas Antineoplásicas) del Servicio de Salud Metropolitano Norte y también pacientes derivados desde las III y IV regiones de Chile, con aproximadamente 90 nuevos casos de cáncer infantil por año. En el hospital se realiza el estudio completo de la patología y se llega a un diagnóstico final. La mayoría de los pacientes se hospitaliza en la Unidad de Oncología; también se pueden ubicar en la Unidad de Paciente Crítico (UPC) y en la Unidad de Pediatría General C (UPG-C) que cuenta con habitaciones de aislamiento.

\section{Diseño del estudio}

Estudio observacional, caso-control, con 2 años de duración, enero de 2012 a diciembre de 2013. A los pacientes con patología oncológica hospitalizados durante ese período en la unidad de oncología, UPC y UPG-C se les realizó estudio de colonización intestinal de ERV mediante un hisopado rectal, según normativa institucional descrita más adelante. Se excluyeron niños con diagnóstico oncológico que no requirieron tratamiento quimioterápico.

Al completar los dos años de estudio, los pacientes se analizaron en dos grupos: casos y controles. Los casos correspondieron a portador de ERV con al menos una muestra positiva durante sus hospitalizaciones y los controles a los no portador de ERV con todas las muestras negativas para ERV durante sus hospitalizaciones. El estudio se basó en los pacientes analizados y no en el número de muestras realizadas.

El punto de comparación en el grupo "portadores de ERV" fue el momento del resultado positivo y en el grupo "no portadores de ERV" el último hisopado rectal negativo tomado durante los dos años de seguimiento.

\section{Toma de muestra}

El hisopado rectal se realizó en pacientes que cumplían con los criterios correspondientes a la normativa institucional vigente de vigilancia de ERV: hospitalización mayor a 5 días de duración, traslado desde otra unidad del hospital o desde otra institución, y en caso de brote, a los contactos que estaban en la misma sala que el caso índice.

La vigilancia epidemiológica se realizó los días miér- coles de cada semana, y en casos de brotes o traslados se realizó el mismo día que ingresaban. Los pacientes fueron estudiados en una o múltiples oportunidades.

Personal de enfermería obtuvo la muestra de hisopado rectal (zona perineo-rectal), la que se recolectó y trasladó en medio de transporte Stuart al laboratorio externo TAAG genetics donde fue procesada.

En las muestras de hisopado rectal se determinó la presencia de ERV por estudio de biología molecular, ya que es un método más sensible y con menor tiempo de respuesta que el cultivo ${ }^{7}$.

\section{Análisis de laboratorio}

Se utilizó la técnica de biología molecular con reacción de polimerasa en cadena (RPC) múltiple usando QuickTAAG ${ }^{\circledR}$ VRE Kit (TAAG genetics, Santiago, Chile). Esta técnica amplifica secuencias objetivo específicas de E. faecium y E. faecalis, además de los genes VanA, VanB, VanC1 y VanC2/C3 e incluye un control positivo interno (ADN plásmido que contiene la secuencia promotora de Arabidopsis thaliana). Se informaron como positivos los resultados con presencia de E. faecium y/o E. faecalis asociado a material genético VanA y/o VanB?

\section{Información clínica}

Se obtuvieron los datos de la ficha clínica en relación a la identificación de posibles FR de colonización de ERV: diagnóstico oncológico, número de días de hospitalización, recuento absoluto de neutrófilos $(\mathrm{RAN})<1.500 / \mathrm{mm}^{3}$ concomitante a la portación de ERV (dentro de las $48 \mathrm{~h}$ previas al estudio), uso de antimicrobianos los 30 días previos, número de hospitalizaciones previas (desde el diagnóstico de cáncer infantil), mucositis (inflamación de mucosas durante los 7 días previos al estudio), cirugías 30 días previos, quimioterapia 14 días previos, episodios de neutropenia febril, estadía en Unidad de Paciente Crítico (UPC), uso de catéter venoso central (CVC) definitivo y detección de Enterococcus spp sensible a vancomicina previo a la detección de ERV.

\section{Análisis estadístico}

Para el análisis descriptivo de las características de los pacientes hospitalizados con patología oncológica se utilizaron porcentajes en el caso de las variables categóricas y para las variables cuantitativas se utilizó la mediana como medida de tendencia central y percentil 25 y 75 como medidas de posición debido a que su distribución no fue simétrica (o Gaussiana). Para determinar la frecuencia de colonización por ERV se utilizó número absoluto y porcentajes, y en el caso del número de días desde el diagnóstico de base a la portación de ERV se utilizó mediana y percentil 25 y 75 . Los factores de riesgo se evaluaron mediante tablas de contingencia, se reportó el odds ratio como medida de riesgo con su intervalo de 
confianza de $95 \%$ y la asociación se evaluó mediante la prueba de chi cuadrado. Para evaluar como FR los diagnósticos oncológicos de los pacientes se utilizó modelos de regresión logística crudo y ajustado por número de días de hospitalización previa, neutropenia concomitante, antimicrobianos 30 días previos y mucositis.

\section{Resultados}

De un total de 107 pacientes se tomaron 422 muestras. Nueve pacientes fueron excluidos, de los cuales cinco fueron trasladados desde o hacia otro centro asistencial y no se les pudo hacer seguimiento completo, y en cuatro fue imposible obtener la ficha clínica. Del total, 98 pacientes fueron evaluables y se incluyeron en el análisis estadístico, encontrándose 51 pacientes colonizados por ERV, lo que indica una frecuencia de colonización de 52\% (Figura 1). Se encontró una diferencia significativa en la frecuencia de portadores de ERV en los años 2012 y 2013 (Figura 2).

La edad al momento de documentar la portación/no portación fue 7,6 y 6,7 años, respectivamente. Un 49\% de los pacientes eran de sexo masculino. Los pacientes con linfoma/tumor sólido representaron $46 \%$ y aquellos con leucemia aguda, tanto linfoide como mieloide, correspondían a $36 \%$. La frecuencia de los diferentes diagnósticos se mantuvo en el grupo de portadores y no portadores de ERV (Tabla 1).

Con respecto a los días transcurridos desde el diagnóstico oncológico y la colonización de ERV: dos de los pacientes se colonizaron precozmente con resultado positivo en la primera muestra tomada, los 49 restantes se colonizaron posteriormente; y el tiempo transcurrido desde el diagnóstico oncológico tiene una mediana de 35 días, con un percentil 25-75 entre 19 y 129 días.

En el análisis univariado de los FR asociados a la portación de ERV, se encontró asociación significativa con el número de días de hospitalización previa, neutropenia concomitante, uso de antimicrobianos 30 días previos y la presencia de mucositis. No se encontró asociación con el número de hospitalizaciones desde el diagnóstico oncológico, episodios de neutropenia febril previos, estadía en UPC, cirugía los 30 días previos, quimioterapia los 14 días previos ni con el uso de CVC definitivo (Tabla 2).

Se evaluó la asociación de portación de ERV con los diagnósticos oncológicos, inicialmente en un análisis univariado, lo que evidenció que el linfoma/tumor sólido es un diagnóstico protector para la portación de ERV y la leucemia mieloide aguda está en el límite de la significancia estadística como FR, con un OR crudo: 0,33 (IC 95\%: 0,14-0,77) y 4,82 (IC 95\%: 0,98-23,6), respectivamente. Sin embargo, al evaluar estas variables en un modelo ajustado por las variables encontradas significativas previamente (Tabla 2) no se mantienen estos resultados.

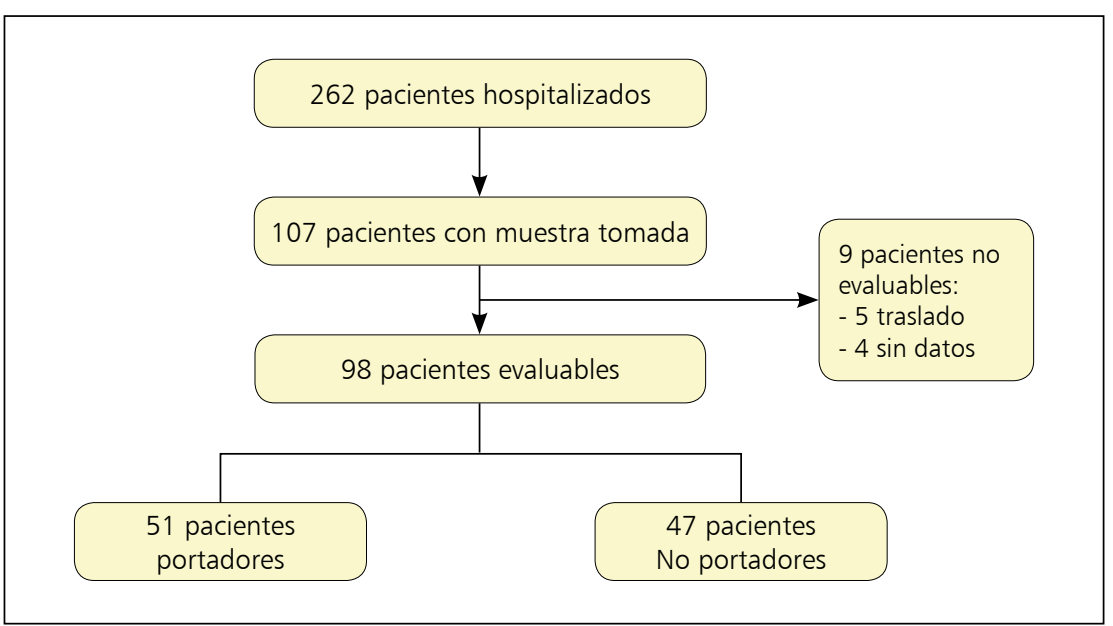

Figura 1. Pacientes portadores y no portadores de ERV durante el período 2012-2013.

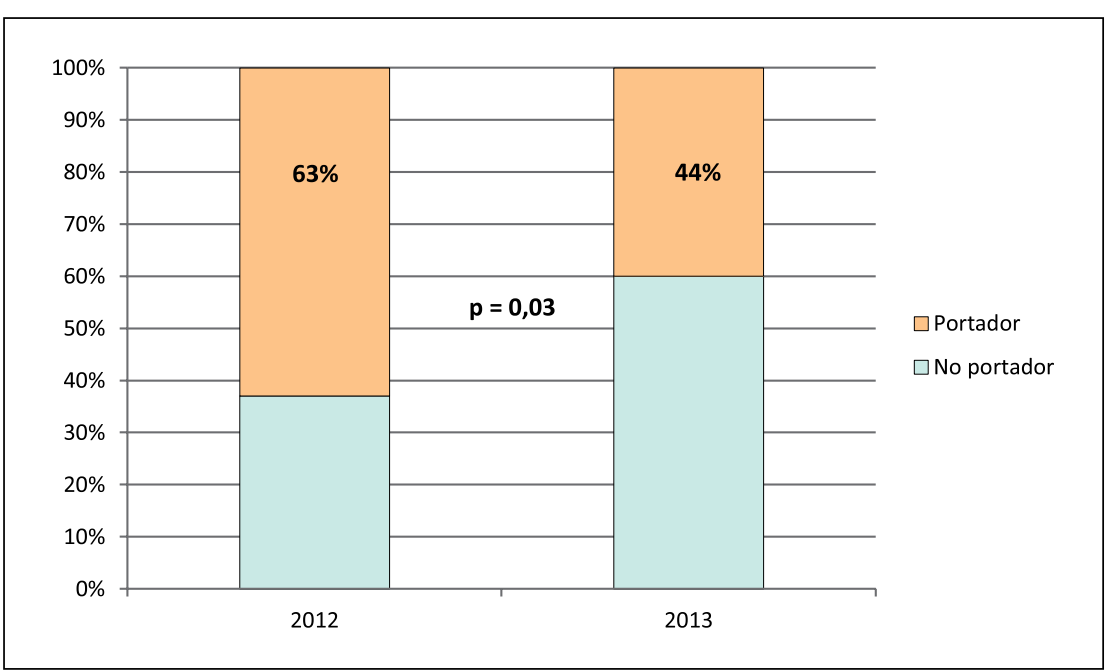

Figura 2. Frecuencia de pacientes portadores de ERV durante los dos años de estudio.

Tabla 1. Características de los pacientes hospitalizados con patología oncológica pediátrica entre los años 2012-2013

$\begin{array}{cccc}\text { Todos } & \text { No portador de ERV } & \text { Portador de ERV } \\ \text { n: } 98 & n: 47 & n: 51\end{array}$

\begin{tabular}{|c|c|c|c|}
\hline Edad* (años) & $6,9(3,2-12,1)^{* *}$ & $6,7(3,2-12,3)^{* *}$ & $7,6(3,2-12,1)^{* *}$ \\
\hline Sexo (\% masculino) & 49 & 53 & 45 \\
\hline \multicolumn{4}{|l|}{ Diagnóstico de base (\%) } \\
\hline LLA & 25 & 17 & 31 \\
\hline LMA & 11 & 4 & 18 \\
\hline Linfoma/Tu sólido & 46 & 60 & 33 \\
\hline Recaída LLA & 7 & 4 & 10 \\
\hline Recaída LMA & 2 & 2 & 2 \\
\hline Recaída Linfoma/Tu Sólido & 9 & 13 & 6 \\
\hline
\end{tabular}

LLA: Leucemia linfoblástica aguda; LMA: Leucemia mieloide aguda; Tu sólido: Tumor sólido. *Edad a la portación. ${ }^{* *}$ Mediana (percentil 25-percentil 75). 


\begin{tabular}{|c|c|c|c|c|}
\hline & $\begin{array}{l}\text { No portador de ERV } \\
\qquad(n=47)\end{array}$ & $\begin{array}{l}\text { Portador de ERV } \\
\quad(n=51)\end{array}$ & Odds Ratio & IC95\% \\
\hline \multicolumn{5}{|l|}{ n hospitalizaciones previas } \\
\hline $1-5$ & 27 & 36 & 1,77 & $0,77-4,09$ \\
\hline $6-10$ & 15 & 11 & 0,58 & $0,23-1,45$ \\
\hline$>10$ & 5 & 4 & 0,71 & $0,18-2,83$ \\
\hline \multicolumn{5}{|l|}{ Días de hospitalización previos } \\
\hline $1-14$ & 43 & 34 & 0,18 & $0,05-0,60^{+}$ \\
\hline $15-30$ & 3 & 11 & 4,03 & $1,04-15,5^{\dagger}$ \\
\hline$>30$ & 1 & 6 & 6,13 & $0,70-52,9$ \\
\hline Neutropenia concomitante* & 11 & 29 & 3,16 & $1,07-9,4^{\dagger}$ \\
\hline - RAN $<500 / \mathrm{mm}^{3}$ & 5 & 18 & 1,96 & $0,38-10,1$ \\
\hline$\geq 3$ episodios neutropenia febril previos & 4 & 12 & 3,31 & $0,89-15,1$ \\
\hline TAM 30 días previos & 19 & 46 & 13,55 & $4,19-50,2^{\dagger}$ \\
\hline$-\geq 4 \mathrm{AM}$ & 7 & 33 & 4,35 & $1,22-15,9^{\dagger}$ \\
\hline Estadía UPC 30 días previos & 14 & 24 & 2,09 & $0,84-5,26$ \\
\hline Usuario CVC definitivo & 34 & 30 & 0,54 & $0,21-1,38$ \\
\hline Mucositis & 1 & 12 & 14,15 & $1,89-617,7^{\dagger}$ \\
\hline Cirugía 30 días previos & 19 & 24 & 1,30 & $0,54-3,15$ \\
\hline Quimioterapia 14 días previos & 33 & 32 & 0,71 & $0,28-1,80$ \\
\hline
\end{tabular}

\section{Tabla 3. Medida de riesgo y asociación entre diagnóstico oncológico y colonización por ERV}

\begin{tabular}{|c|c|c|c|c|c|c|}
\hline Diagnóstico de base & $\mathbf{n}$ & $\begin{array}{c}\text { Portador de ERV } \\
\%\end{array}$ & $\begin{array}{c}\text { Odds Ratio } \\
\text { crudo }\end{array}$ & $\mathrm{IC} 95 \%$ & $\begin{array}{l}\text { Odds Ratio } \\
\text { Ajustado* }\end{array}$ & IC95\% \\
\hline Linfoma/Tu sólido & 45 & 38 & 0,33 & $0,14-0,77$ & 0,59 & $0,16-2,19$ \\
\hline LLA & 24 & 67 & 2,22 & $0,85-5,84$ & 1,45 & $0,35-5,93$ \\
\hline LMA & 11 & 82 & 4,82 & $0,98-23,6$ & 2,28 & $0,22-23,5$ \\
\hline Recaída LLA & 7 & 71 & 2,44 & $0,45-13,2$ & 1,04 & $0,08-12,3$ \\
\hline Recaída LMA & 2 & 50 & 0,92 & $0,05-15,1$ & 0,13 & $0,01-3,03$ \\
\hline Recaída linfoma/Tu sólido & 9 & 33 & 0,42 & $0,10-1,81$ & 1,46 & $0,50-42,1$ \\
\hline
\end{tabular}

Se presentan los resultados del modelo logístico ajustado según el R cuadrado ajustado en Tabla 3.

\section{Discusión}

En nuestro conocimiento, esta es la primera investigación que se realiza en nuestro país en pacientes pediátricos con patología oncológica y que utiliza la técnica de RPC múltiple de ERV. Esta técnica es más sensible que los métodos tradicionales ${ }^{7}$, lo que puede permitirnos caracterizar mejor los FR de colonización por este microorganismo. Además, las técnicas de diagnóstico molecular pueden aportar con tiempo de respuesta más rápido que los cultivos tradicionales, permitiendo tomar decisiones clínicas más rápido en relación al aislamiento y gestionar mejor la ocupación de las camas hospitalarias disponibles.

Durante el período de estudio, se observó que 52\% de los pacientes se colonizaron con ERV, cifra superior al $18-25 \%$ reportado en la literatura médica ${ }^{1,8}$, lo que puede explicarse por la alta sensibilidad de la RPC múltiple $(98 \%)$ comparada con el cultivo $(70 \%)$ y la frecuencia con 
que se tomaron las muestras ${ }^{7}$. La disminución significativa de portadores desde $63 \%$ el año 2012 a $44 \%$ el 2013, puede deberse a la intervención realizada en la Unidad de Oncología, con educación a los padres, al personal de salud y modificación de las normas de la unidad para prevenir la propagación de la colonización por esta bacteria.

En la descripción de los pacientes se observa que $36 \%$ de ellos presentaba leucemia aguda, concordante con la literatura científica publicada que sitúa a las leucemias con una frecuencia alrededor de 35 a $40 \%$ de todos los cánceres en niños 9 .

La mitad de los portadores de ERV se colonizó durante los primeros 35 días desde su diagnóstico, dos antes de ello, lo que señala que la educación y prevención de la colonización por ERV debe hacerse desde el primer día de hospitalización.

La presencia de mucositis fue el FR encontrado con mayor asociación a la colonización de ERV, esto podría explicarse por la pérdida de esta barrera natural, permitiendo el contacto y la permanencia de ERV en la mucosa intestinal. La prevención y tratamiento de la mucositis puede ser un elemento fundamental para mantener esta barrera indemne y así disminuir el riesgo de portación de este agente.

El uso y la cantidad de antimicrobianos, cuatro o más, durante los 30 días previos a la pesquisa de ERV, fue otro FR significativo en nuestro estudio. Por ello se debe tener especial consideración en el tratamiento de las infecciones de estos pacientes, ya que se debe cubrir todo posible foco de infección, pero no agregar antimicrobianos sin fundamento, ya que se está favoreciendo la colonización por ERV, aumentando el riesgo de infección por este agente $^{6}$. El elevado número de antimicrobianos utilizado en pacientes oncológicos pediátricos es una inquietud que ha surgido dentro del grupo PINDA, por lo cual se encuentra en marcha un trabajo de investigación Fondecyt (Fondo Nacional de Desarrollo Científico y Tecnológico) que aborda el uso racional de antimicrobianos, el cual nos permitirá tener un enfrentamiento más adecuado frente a las infecciones.

La neutropenia concomitante, RAN $<1.500 / \mathrm{mm}^{3}$, también evidenció mayor asociación a la colonización por esta bacteria. Esto se explicaría por la disminución de uno de los primeros mecanismos de defensa frente a microorganismos patógenos. Por ello cobra importancia conocer y aplicar medidas de prevención en estos pacientes, como son el lavado de manos frecuente, el uso de guantes y pechera. Un elemento a destacar es que el RAN $<500 / \mathrm{mm}^{3}$ no presentó mayor riesgo de colonización por ERV. Esto puede deberse a un bajo poder de la muestra, por lo que se deja abierta la pregunta para futuros trabajos de investigación.

El número de días de hospitalización previos a la pesquisa de ERV también fue encontrado como FR. Esto se debería a la exposición a microorganismos intrahospitalarios resistentes a antimicrobianos y es concordante con publicaciones previas ${ }^{3}$.

En nuestro centro asistencial, la mayoría de los pacientes con leucemia mieloide aguda estaban colonizados por ERV; sin embargo, no se encontró asociación significativa para la portación con este diagnóstico. Se puede inferir entonces, que la neutropenia secundaria a los distintos esquemas de quimioterapia, el uso de tratamiento antimicrobiano prolongado, entre otros, son los elementos asociados a la colonización por ERV, más que el diagnóstico oncológico propiamente tal.

Este trabajo permitió determinar FR asociados a la colonización por ERV en pacientes oncológicos pediátricos y proponer algunas medidas de prevención que pueden ser efectivas acorde a lo encontrado. Sin embargo, tiene la limitación de ser un trabajo con un número de pacientes acotado. Otra limitación es no poder objetivar si nuestros pacientes tuvieron contacto directo o no con pacientes colonizados por ERV, ya que este dato no lo consignamos específicamente y es un mecanismo conocido de diseminación de este microorganismo. Sin embargo, con estos resultados se puede plantear futuros estudios que aborden estos FR con diseño prospectivo y multicéntrico, y así validar los FR encontrados.

Finalmente, concluimos que existen múltiples FR, y que frente a esto también podemos tomar múltiples medidas para prevenir la colonización por ERV, con lo que esperamos que este trabajo sea de ayuda en la tarea de controlar la propagación de microorganismos multiresistentes entre nuestros pacientes.

Agradecimientos. Nuestros más sinceros agradecimientos a EU. Fanny Sepúlveda, enfermera Jefe de la Unidad de Oncología, ya que sin su ayuda este trabajo no habría sido posible de realizar.

\section{Resumen}

Introducción: El aislamiento de Enterococcus spp resistentes a vancomicina (ERV) ha presentado un incremento significativo en los últimos años, aumentando el riesgo de infección por esta bacteria y favoreciendo su diseminación. Nuestro objetivo es determinar los factores de riesgo (FR) de colonización intestinal de ERV en pacientes oncológicos internados en el Hospital de Niños Roberto del Río. Método: Entre enero de 2012 y diciembre de 2013 se realizó un estudio transversal de colonización rectal por ERV mediante muestras de hisopado rectal obtenidas en 107 pacientes efectuando RPC múltiple para ERV. Se dividió en grupo "portador" y "no portador" y se evaluó los posibles FR para colonización por ERV. Resultados: Se encontró colonización por ERV en 51 pacientes (52\%). El tiempo transcurrido desde el diagnóstico oncológico 
y la colonización presentó una mediana de 35 días. Los FR encontrados con asociación significativa fueron el número de días de hospitalización previa, neutropenia, uso de antimicrobianos 30 días previos y mucositis. Conclusión: De acuerdo a los FR encontrados podemos sugerir medidas de prevención para colonización por ERV. Esta es la primera investigación realizada en nuestro país en pacientes oncológicos pediátricos y que utiliza la técnica de RPC múltiple para ERV, lo que permite un aporte significativo sobre este tema en Chile.

\section{Referencias bibliográficas}

1.- Nateghian A, Robinson J L, Arjmandi K, Vosough P, Karimi A, et al. Epidemiology of vancomycin-resistant enterococci in children with acute lymphoblastic leukemia at two referral centers in Tehran, Iran: a descriptive study. Int J Infect Dis 2011; 15: e332-5.

2.- Han S H, Chin B S, Lee H S, Jeong S J, Choi H K, Kim C O, et al. Vancomycinresistant enterococci bacteremia: Risk factors for mortality and influence of antimicrobial therapy on clinical outcome. J Infect 2009; 58: 182-90.

3.- Braun S, Fica A, Andrighetti D, Porte L, Triantafilo V. Estudio de factores de riesgo para colonización por enterococo resistente a vancomicina en el Hospital Militar de Santiago. Rev Chilena Infectol 2009; 26 (4): 374-5.

4.- Tschudin Sutter S, Frei R, Dangel M, Gratwohl A, Bonten M, Widmer A F. Not all patients with vancomycin-resistant enterococci need to be isolated. Clin Infect Dis 2010; 51 (6): 678-83.

5.- Hélio S. Sander: Enterococos resistentes a vancomicina: ¿Infección emergente inminente? Rev Chilena Infectol 2002; v.19: supl 1: S50-5.

6.- Iosifidis E, Karakoula K, Protonotariou E, Kaperoni M, Matapa E, Pournaras S, et al. Polyclonal outbreak of vancomycin-resistant Enterococcus faecium in Pediatric Oncology Department. J Pediatr Hematol Oncol 2012; 34 (7): 511-6.
7.- Benadof D, San Martín M, Aguirre J, Paredes L, Malig R, Melo F, et al. A new multiplex PCR assay for the simultaneous detection of vancomycin-resistant enterococci from rectal swabs. J Infect 2010; 60 (5): 354-9.

8.- Reale A, Depetri M, Culasso C, Paviolo M, Cheguirián M L, Enrico M C, et al. Enterococos resistentes a vancomicina: prevalencia $\mathrm{y}$ factores asociados a la colonización intestinal en pacientes oncológicos del Hospital de Niños de Córdoba. Rev Arg Microb 2009; 41: 92-6.

9.- Guía Clínica Leucemia en menores de 15 años. Series guías clínicas MINSAL 2010, Santiago, Chile. web.minsal.cl/portal/url/item/7220fdc433 e944a9e04001011f0113b9.pdf 